\title{
Multichannel Quantum Defect Study of the Perturber's Effect on the Overlapping Resonances in Rydberg Series for the Systems Involving 2 Closed and Many Open Channels
}

\author{
Chun-Woo Lee \\ Department of Chemistry, Ajou University, Suwon 443-749, Korea.E-mail: clee@ajou.ac.kr \\ Received February 14, 2010, Accepted March 31, 2010
}

\begin{abstract}
The phase-shifted version of the multichannel quantum-defect theory (MQDT) was reformulated by disentangling the interloper spectrum from the perturbed dense Rydberg series for a systems involving 2 closed and more than 1 open channel. The theory was applied successfully to Martins and Zimmermann's photoionization spectra of the Rydberg series $\mathrm{Cu}$ I $3 d^{9} 4 s\left({ }^{1} D_{2}\right) n d^{2} G_{9 / 2}$ perturbed by the interloper, $3 d^{9} 4 p^{24} F_{9 / 2}$, for which Cohen's 4 channel QDT had failed. The zero surface graphic of the perturbed Fano's asymmetry parameter $q$ of the autoionization spectrum of dense Rydberg series by the interloper was determined by only two parameters for this system. It was used as a map to trace the transformation route of the 3 channel autoionization spectra to the 4 channel spectra when the channel coupling of the closed channels with a newly added open channel was turned on progressively.
\end{abstract}

Key Words: Phase-shifted MQDT, Overlapping resonance, 4 Channel QDT

\section{Introduction}

Complex overlapping resonances caused by an interloper are observed routinely in autoionization spectra. ${ }^{1}$ Multichannel quantum defect theory (MQDT), with the implementation of phase renormalization, is extremely powerful and used routinely to deal with the observed overlapping resonances with only a few parameters. ${ }^{2}$ Although MQDT yields a few parameters that reproduce the observed spectra, the nature of the channel couplings is obscure with the simple use of theory. In previous work, ${ }^{3}$ the analysis tool was developed to reveal the nature of the channel coupling for the overlapping resonances in the systems, particularly for the perturbed Rydberg series with a single closed channel acting as an interloper using 3 channel QDT with one open and 2 closed channels (abbreviated as a 3 channel QDT). Although the 3 channel QDT can reproduce the numerous overlapping resonances observed, more channels are frequently needed. Some people suggest that each closed channel requires each effective open channel so that it is imperative to add more open channels. ${ }^{4}$ However, thus far, it is unclear how additional open channels will affect channel coupling. In this respect, the theory does not appear to have developed a great deal from Fano and Cooper's recognition, ${ }^{5}$ in that a discrete state can interact with only one type of continuum, and from Mies's proposal ${ }^{6}$ to use an overlapping matrix to represent the extent of overlap when a complete superimposition of overlapping resonances is no longer obtained when more than 1 open channel is involved in configuration mixing (CM) theory. Mies's CM theory was implemented into MQDT for the treatment of the spectral width in isolated core excitation (ICE) spectra by Lecomte. ${ }^{7}$ Cohen challenged this problem of handling the channel coupling induced by additional open channels with the approximate analytic solution but was only partially successful. ${ }^{8}$ His theory satisfactorily handled the experimental spectra of $\mathrm{Ba}$, where direct closed channel coupling is stronger than the indirect one through the continuum. On the other hand, it failed for the experimental spectra of $\mathrm{Cu}$, where the direct closed channel interaction is negligible.

In previous work, ${ }^{3}$ it was reported that Ueda's formalism ${ }^{9}$ of separating the interloper's spectra from the one of the perturbed Rydberg series in a 3 channel QDT provides a very useful analysis tool for overlapping resonance spectra when the poles of the parameters are handled well and the zero surface structures of the line asymmetry parameter $q$ are clearly revealed. Therefore, it is natural to extend this separation of the interloper's spectra from the perturbed Rydberg one and explore zero and pole structures for systems involving more than 1 open channel and to determine the role of an additional open channels on channel coupling and the spectra.

Reformulation of the photoionization cross section formula into the Ueda's type is liable to the contamination of Fano's asymmetry parameter $q$ with the poles, and may not be useful. The adoption of Cohen's choice for the eigenchannels will be shown to reduce this difficulty considerably. He suggested using the most trouble free formula for the eigenchannels whereby the eigenchannels can be made into resonant and nonresonant ones. With this choice of eigenchannels, troublesome poles can be driven into non-resonant eigenchannels leaving only kinks in the resonant eigenchannels.

This theory was applied to Martins and Zimmermann's strange-looking photoionization spectra ${ }^{10}$ of the Rydberg series $\mathrm{Cu}$ I $3 d^{9} 4 s\left({ }^{1} D_{2}\right) n d^{2} G_{9 / 2}(n=16-20)$ perturbed by the interloper $3 d^{9} 4 p^{2}{ }^{4} F_{9 / 2}$ for which Cohen's 4 channel QDT failed.

\section{Brief Introduction of MQDT}

In the multichannel quantum defect theory of the photoionization process, the coordinate $R$ for the relative motion of colliding partners along which ionization occurs is divided into two ranges $R \leq R_{0}$ and $R>R_{0}$, the inner and outer ones, respectively. In contrast to the inner range, where transfers in energy, momentum, angular momentum, spin, or the formation of a tran- 
sient complex occur due to the strong interactions, channels are decoupled in an outer range. Consequently, the motion is governed by ordinary second-order differential equations and can be described by a superposition of the energy-normalized regular and irregular base pair $f_{j}(R)$ and $g_{j}(R)$ whose analytical forms are known and specified by the long-range quantum defect theory (qdt) parameters $\eta_{j}$ and $\beta_{j}$. Using these pairs, $N$ independent solutions of the Schrödinger equation for the decoupled motion in the intermediate range $\left(R>R_{0}\right)$ for an $N$-channel system in the energy range of autoionization can be expressed as a standing-wave type $\Psi_{i}(R, \omega)=\sum_{j=1}^{N} \Phi_{j}(\omega)\left[f_{j}(R) \delta_{j i}-\right.$ $\left.g_{j}(R) K_{j i}\right]$ where $\Phi_{j}(\omega)$ are the channel basis functions for the coordinate space excluding $R$ and $K_{j i}$ is called short-range reactance matrix containing the information on channel coupling. Note that they are degenerate in the autoionization regime. The complex dynamics occurring in the strongly coupled inner region affects the dynamics in the outer region and subsequently the photoionization spectra only through the energy insensitive short-range reactance matrix $K_{j i}$. Although all the $N$ solutions are needed to describe the motion in the intermediate range, some become closed and no longer exist in the limit of $R \rightarrow \infty$. The notations $P$ and $Q$ were used for the sets of open and closed channels, respectively and the super-indices $o$ and $c$ were used for dynamic quantities belonging to the open and closed channels, respectively. Note that classification of these channels as open or closed is meaningful only at a large $R$. Nonetheless, it may still be convenient to keep this classification in the intermediate range. In this practice, $K$ may be decomposed into the submatrices as $\left[K^{c c} K^{c o} ; K^{o c} K^{o o}\right]$.

Strong energy dependence of photoionization spectra observed around resonance enters in MQDT when the boundary conditions at $R \rightarrow \infty$ are applied. The equations obtained by the application of boundary conditions are called compatibility equations or MQDT equations. Decoupled nature of the asymptotic region allows analytical treatment of the compatibility equations. Note that the equations contain only energy-insensitive short-range reactance matrix $K$ and long-range qdt parameters, indicating that photoionization spectra can be described with only a few energy insensitive parameters.

Although MQDT described so far is powerful enough to describe the photoionization spectra with a few energy-insensitive parameters, it became more powerful by further development by Giusti-Suzor and Fano ${ }^{11}$ and Cooke and Cromer ${ }^{12}$ independently. They utilized the degree of freedom in choosing the base pairs $\left(f_{j}, g_{j}\right)$ to find the phase-shifted base pair so that $K^{o o}$ becomes a null matrix and $K^{c c}$ becomes either a null matrix or a matrix with null-diagonal, depending on the system. The representation in these phase-shifted base pairs will be called the tilde representation and will be denoted with tilde.

We will use this phase-shifted version of MQDT in the next two sections to reformulate the photoionization cross section formula into the Ueda's type ${ }^{9}$ when more than 1 open channels are involved. The reformulation starts from the introduction of resonance parameters which are intrinsic to resonance scattering independent of the preparation and detection processes. By this, avoided crossing interaction which is the other part of induced dynamics of resonance scattering can be separately described from the part due to the intrinsic resonance scattering. Reformulation into the Ueda's type can then be performed.

Introduction of resonance parameters. Currently, the practical use of multichannel quantum defect theory (MQDT) almost always adopts the phase-shifted MQDT and begins directly from either zero submatrices or zero diagonal submatrices depending on the type of system. ${ }^{13}$ According to this practice, by the suitable phase renormalization described in Ref [7], the short-range reactance matrix for systems involving 2 closed and many open channels can be made to the following form:

$$
\tilde{K}=\left(\begin{array}{cc}
\tilde{K}^{c c} & \tilde{K}^{c o} \\
\tilde{K}^{o c} & \tilde{K}^{o o}
\end{array}\right)=\left(\begin{array}{ccccc}
0 & \tilde{K}_{12} & \tilde{K}_{13} & \cdots & \tilde{K}_{1 n} \\
\tilde{K}_{12} & 0 & \tilde{K}_{23} & \cdots & \tilde{K}_{2 n} \\
\tilde{K}_{13} & \tilde{K}_{23} & 0 & \cdots & 0 \\
\vdots & \vdots & \vdots & \ddots & \vdots \\
\tilde{K}_{1 n} & \tilde{K}_{2 n} & 0 & \cdots & 0
\end{array}\right)
$$

where indices 1 and 2 will be used for the closed channel and higher indices will be used for the open ones. The ionization thresholds $I_{i}$ are assumed to satisfy $I_{3}<\cdots<I_{n}<I_{1}<I_{2}$. Form (1) indicates that direct channel coupling is possible either between closed channels 1 and 2 or between closed and open channels in the tilde representation. If it were not for nonzero $\tilde{K}_{12}$, only channel couplings between closed and open channels would be retained, indicating that couplings causing background scattering are removed and the ones causing resonance scattering are retained.

Autoionizing states that yield the photoionization spectra can be described by the linear combination of the eigenchannel wavefunctions $\Psi_{\rho}^{(e)}$ which diagonalize the physical reactance matrix $\tilde{\mathbf{K}}$ given in terms of the submatrices of $\tilde{K}$ of Eq. (1) as $\tilde{K}^{o o}-\tilde{K}^{o c}\left(\tan \tilde{\beta}+\tilde{K}^{c c}\right)^{-1} \tilde{K}^{c o}$. Let us expand this energy-normalized wavefunction $\Psi_{\rho}^{(e)}$ belonging to eigenchannel $\rho$ to

$$
\tilde{\Psi}_{\rho}^{(e)}=\sum_{i \in P} \tilde{\Psi}_{i} \tilde{Z}_{i \rho} \cos \tilde{\delta}_{\rho}+\sum_{i \in Q} \tilde{\Psi}_{i} \cos \pi\left(v_{i}+\mu_{i}\right) \tilde{Z}_{i \rho}
$$

in terms of the standing-wave channel basis functions in open channel space $P$ and closed channel space $Q$. The superscript (e) stands for eigenchannel. The additional factors $\cos \tilde{\delta}_{\rho}$ and $\cos \pi\left(v_{i}+\mu_{i}\right)$ besides expansion coefficients are introduced to make $\tilde{Z}_{i \rho}(i \in P)$ unitary and needed since the standingwaves $\tilde{\Psi}_{i}$ defined by Eq. (3) are not energy-normalized. $\tilde{\Psi}_{i}$ can be decoupled outside the reaction zone as follows:

$$
\tilde{\Psi}_{i}=\sum_{j=1}^{n} \Phi_{j}(\omega)\left[\tilde{f}_{j}(R) \delta_{j i}-\tilde{g}_{j}(R) \tilde{K}_{j i}\right], \quad\left(R>R_{0}\right),
$$

where $\left(\tilde{f}_{j}, \tilde{g}_{j}\right)$ is the phase-shifted regular and irregular base pair $^{11}$

$$
\begin{aligned}
& \tilde{f}_{j}=f_{j} \cos \pi \mu_{j}-g_{j} \sin \pi \mu_{j} \\
& \tilde{g}_{j}=g_{j} \cos \pi \mu_{j}+f_{j} \sin \pi \mu_{j} .
\end{aligned}
$$


The regular and irregular base pair $\left(f_{j}, g_{j}\right)$ belong to the $j^{\text {th }}$ ionization threshold energy $I_{j}$ with an effective quantum number $v_{j}$ given by $E=I_{j}-\mathrm{Ryd} / v_{j}^{2}$, where Ryd denotes Rydberg constant. As is well known, ${ }^{2}$ the expansion coefficients into the closed channel basis functions in (2) can be obtained as follows:

$$
\cos \tilde{\beta} \tilde{Z}_{i \rho}^{c}=-\left[\left(\tan \tilde{\beta}+\tilde{K}^{c c}\right)^{-1} \tilde{K}^{c o}\right]_{i \rho} \tilde{Z}_{\rho}^{o} \cos \tilde{\delta}_{\rho},
$$

where $\tilde{\beta}_{i}$ is $\pi\left(v_{i}+\mu_{i}\right)$ and $\tilde{\delta}_{\rho}(\rho \in P)$ is the eigenphase shift obtained from the compatibility equation

$$
\operatorname{det}\left(\tilde{\mathbf{K}}-\tan \tilde{\delta}_{\rho}\right)=0
$$

for the MQDT equation satisfied by the unit column vector $\tilde{\mathbf{Z}}_{\rho}^{o}=\left\{\tilde{Z}_{i \rho}^{o} \mid i \in P\right\}$

$$
\left(\begin{array}{cc}
\tilde{K}^{c c}+\tan \tilde{\beta} & \tilde{K}^{c o} \\
\tilde{K}^{o c} & -\tan \tilde{\delta}_{\rho}
\end{array}\right)\left(\begin{array}{c}
\tilde{\mathbf{Z}}_{\rho}^{c} \\
\tilde{\mathbf{Z}}_{\rho}^{o}
\end{array}\right)=0,
$$

where $\tilde{K}^{o o}=0$ is used. The expansion coefficients $\tilde{Z}_{i \rho}^{o}$ for open channel space $P$ in Eq. (2) is obtained by solving Eq. (6).

If more than one open channel is involved, avoided crossing interactions between the eigenphase shifts can occur, which will obscure the resonance behavior. Removal of the avoided crossing interactions can be achieved easily by considering a sum of the eigenphase shifts. The removal of avoided crossing interactions introduces parameters that only characterize the resonance behavior and have little to do with the avoided crossing interactions. This will help disentangle the resonant scattering from the avoided crossing interactions from the photoionization cross section spectra.

The eigenphase sum is obtained easily from the determinant of the scattering matrix ${ }^{14}$ as follows

$$
\begin{aligned}
\operatorname{det}(\tilde{\boldsymbol{S}}) & =\exp \left(-2 i \sum_{j} \tilde{\delta}_{j}\right) \\
& =\frac{\operatorname{det}\left(\tan \tilde{\beta}+\tilde{\kappa}^{c c^{*}}\right)}{\operatorname{det}\left(\tan \tilde{\beta}+\tilde{\kappa}^{c c}\right)}=\exp \left(-2 i \tilde{\delta}_{r}\right)
\end{aligned}
$$

where $\tilde{\kappa}^{c c}$ denotes $\tilde{K}^{c c}-i \tilde{K}^{c o}\left(1+i \tilde{K}^{o o}\right)^{-1} \tilde{K}^{o c}=\tilde{K}^{c c}-i \tilde{K}^{c o} \tilde{K}^{o c}{ }^{7}$. $\Sigma_{j} \tilde{\delta}_{j}^{0}=0$ from $\tilde{K}^{o o}=0$ in the phase-shifted MQDT yields the $3^{\text {rd }}$ equality in Eq. (8). The resonant phase shift $\tilde{\delta}_{r}$, which was originally defined as the phase of $\operatorname{det}\left(\tan \tilde{\beta}+\tilde{\kappa}^{c c}\right)$, is obtained as follows:

$$
\tan \tilde{\delta}_{r}=-\frac{\tilde{\xi}_{1}^{2} \tan \tilde{\beta}_{2}+\tilde{\xi}_{2}^{2} \tan \tilde{\beta}_{1}-2 \tilde{K}_{12} \tilde{\xi}_{1} \cdot \tilde{\xi}_{2}}{\tan \tilde{\beta}_{1} \tan \tilde{\beta}_{2}-\tilde{K}_{12}^{2}-\tilde{\xi}_{1}^{2} \tilde{\xi}_{2}^{2} \sin ^{2} \theta}
$$

where $\tilde{\xi}_{i}$ denotes the modulus of the column vector $\tilde{\xi}_{i}$ whose $k^{\text {th }}$ component is defined as $\tilde{K}_{k i}^{o c}$ and stands for the coupling streng- th of closed channel $i$ with open channels. Likewise, $\tilde{\xi}_{1} \cdot \tilde{\xi}_{2} \underset{\sim}{\text { de- }}$ notes the scalar product of two vectors defined by $\sum_{k \in P} \tilde{K}_{k 1} \tilde{K}_{k 2}$ and stands for the indirect coupling between closed channels 1 and 2. It can be written in matrix form as $\tilde{\xi}_{1}^{T} \tilde{\xi}_{2}$. The square of the modulus of $\operatorname{det}\left(\tan \tilde{\beta}+\tilde{\kappa}^{c c}\right)$ is greatly simplified as follows:

$$
C^{2}=\tilde{W}_{1 e f f}^{2} \tilde{W}_{2}^{2}\left(\tilde{\varepsilon}_{1 e f f}^{2}+1\right)\left(\tilde{\varepsilon}_{2}^{2}+1\right),
$$

where $\tilde{\varepsilon}_{i}$ denotes $\tan \tilde{\beta}_{i} / \tilde{W}_{i}(i=1,2)$ and $\tilde{W}_{i}$ denotes $\tilde{\xi}_{i}^{2}$ and stand for the reduced energy and spectral width for Rydberg series $i$, respectively. The form is the same as the one for the 3 channel system involving 1 open and 2 closed channels ${ }^{3}$ but the effective reduced spectral width and energy parameters $\tilde{W}_{\text {leff }}$ and $\tilde{\varepsilon}_{1 \text { eff }}$ for Rydberg series 1 include additional terms containing $\sin ^{2} \theta$ to the ones for the 3 channel system:

$$
\begin{aligned}
\tilde{W}_{1 e f f} & =\tilde{W}_{1}\left[\sin ^{2} \theta+\cos ^{2} \theta \frac{\left(\tilde{\varepsilon}_{2}+\tilde{q}_{W}\right)^{2}}{\tilde{\varepsilon}_{2}^{2}+1}\right], \\
\tilde{\varepsilon}_{\text {leff }} & =\frac{\tan \tilde{\beta}_{1}}{\tilde{W}_{1 \text { eff }}}+\frac{\left[\tilde{\varepsilon}_{2}\left(1-\tilde{q}_{W}^{2}\right)+2 \tilde{q}_{W}\right] \cos ^{2} \theta}{\left(\tilde{\varepsilon}_{2}+\tilde{q}_{W}\right)^{2} \cos ^{2} \theta+\left(\tilde{\varepsilon}_{2}^{2}+1\right) \sin ^{2} \theta} \\
& =\frac{\tilde{W}_{1}}{\tilde{W}_{\text {leff }}}\left[\tilde{\varepsilon}_{1}+\cos ^{2} \theta \frac{\tilde{\varepsilon}_{2}\left(1-\tilde{q}_{W}^{2}\right)+2 \tilde{q}_{W}}{\tilde{\varepsilon}_{2}^{2}+1}\right],
\end{aligned}
$$

where $\tilde{q}_{W}$ is also the generalization of $-\tilde{K}_{12} /\left(\tilde{\xi}_{1} \tilde{\xi}_{2}\right)$ in the 3 channel QDT involving 1 open and 2 closed channels and is defined as follows:

$$
\tilde{q}_{W}=-\frac{\tilde{K}_{12}}{\tilde{\xi}_{1} \cdot \tilde{\xi}_{2}}=-\frac{\tilde{K}_{12}}{\tilde{\xi}_{1} \tilde{\xi}_{2} \cos \theta} .
$$

It stands for the ratio between the direct and indirect couplings between closed channels 1 and 2. Note that the couplings parameters $\tilde{\xi}_{i}$ between a closed channel $i$ and an open channel becomes the vector $\tilde{\xi}_{i}$ in open channel space $P$ with the component $\tilde{K}_{k i}^{o c}(k \in P)$ when more than 1 open channel is involved. Since $\tilde{\xi}_{1}$ and $\tilde{\xi}_{2}$ are vectors in open space $P$, they can point in different directions and the angle $\theta$ they form is not zero in general. Note that parallel or anti-parallel directions are only allowed for 1 open channel system. ${ }^{6}$ Eqs. (11), (12) and (13) revert to the corresponding equations for the 3 channel QDT involving 1 open and 2 closed channels when $\theta=0$.

Mies first considered this problem in the context of configuration mixing theory and concluded that the completely superimposable $\theta=0$ case corresponds to an isolated resonance. ${ }^{6}$ The same problem was also considered in MQDT by Lecomte. ${ }^{7}$ Mies's conclusion is equally applicable to the present case of MQDT. Eq. (11) shows that the spectral width $\tilde{W}_{\text {leff }}$, which was studied extensively by Cooke and Cromer ${ }^{12}$ and by Lecomte ${ }^{7}$ to explain the isolated core excitation(ICE) spectra and is identical 
to the most general form of the spectral width obtained by Lecomte, is composed of an incoherent sum of contributions from isolated and overlapping resonances.

Separation of the spectrum of a perturber from the dense Rydberg spectra. A Rydberg series perturbed by an interloper is an important system showing complex resonance phenomena due to the overlapping resonances. The reformulation of the phaseshifted MQDT by Ueda involving 2 closed and 1 open channel to disentangle the interloper spectra from the perturbed dense Rydberg series was used extensively in previous work to determine the relative influences between the interloper and dense Rydberg series caused by the overlapping of resonances. The followings give a few of the items obtained by the disentanglement: the role of the spectrum of an interloper as an envelope and being served as a structured background; the huge intensity borrowing of the lines of dense Rydberg series from the interloper taking place at the simple poles; and construction of the zero surfaces of the line profile index $\tilde{q}_{\text {leff }}$ for use as a compass to find interesting overlapping resonance phenomenon, such as straddling intervening resonance. However, during the process of disentanglement, singularities enter and obstruct the analysis. The result of his formulation could be harvested only by careful analysis of the singularities and zeros of his formulation, as done in Ref. [3].

Let us extend the previous formulation to the system involving more than 1 open channel. In the formulation, it is important to emphasize the role of additional open channels comparing the 3 channel QDT involving 2 closed and 1 open channel. This can be achieved by treating each eigenchannel and 2 closed channels as consisting of a 3 channel system and applying Ueda's formulation to each of these 3 channel systems. The total photoionization cross section can then be obtained as a sum of the cross sections of the 3 channel systems belonging to each eigenchannel.

From a theoretical point of view, this is not the desired approach because the eigenchannels are not directly observable. A better approach might be to apply density matrix formalism that includes only the observed quantities. Undesirable Ueda type's approach was found to be better for the present work because it handles the interference between the closed and open channels directly and obtains the pole and zero structures of the spectral asymmetry parameter $q$, which is related to the extent of the interference between the closed and open channels.

The eigenchannels can be divided into resonant and nonresonant ones as reported by Cohen. ${ }^{8}$ The restriction of det $\left(\tan \tilde{\beta}+K^{c c}\right)=0$, which Cohen employed as an approximation, is not accepted here. In this study, the only restriction is the number of closed channels, 2 .

Substitution of Eq. (5) into (2) yields the eigenchannel basis functions in matrix form as follows:

$$
\tilde{\Psi}_{\rho}^{(e)}=\left[\tilde{\Psi}^{o}-\tilde{\Psi}^{c}\left(\tan \tilde{\beta}+\tilde{K}^{c c}\right)^{-1} \tilde{K}^{c o}\right] \tilde{\mathbf{Z}}_{\rho}^{o} \cos \tilde{\delta}_{\rho}
$$

The corresponding transition dipole moment is given in matrix form by

$$
\tilde{D}_{\rho}^{(e)}=\left[\tilde{D}^{o}-\tilde{D}^{c}\left(\tan \tilde{\beta}+\tilde{K}^{c c}\right)^{-1} \tilde{K}^{c o}\right] \tilde{\mathbf{Z}}_{\rho}^{o} \cos \tilde{\delta}_{\rho} .
$$

It is convenient to consider the $0^{\text {th }}$ order eigenchannel basis function composed of the open channel component alone:

$$
\tilde{\psi}_{\rho}^{(e)}=\sum_{i \in P} \tilde{\Psi}_{i} \tilde{Z}_{i \rho}^{o}
$$

and the corresponding transition dipole moment

$$
\tilde{d}_{\rho}^{(e)}=\sum_{i \in P} \tilde{D}_{i} \tilde{Z}_{i \rho}^{o}
$$

The closed channel part can be transformed in matrix form to

$$
\begin{aligned}
\tilde{D}^{c}\left(\tan \tilde{\beta}+\tilde{K}^{c c}\right)^{-1} \tilde{K}^{c o} \tilde{\mathbf{Z}}_{\rho}^{o} \\
=\frac{1}{D_{c c}}\left[\tilde{D}_{1}\left(\tilde{T}_{2} \tilde{\xi}_{1}^{T} \tilde{\mathbf{Z}}_{\rho}^{o}-\tilde{K}_{12} \tilde{\xi}_{2}^{T} \tilde{\mathbf{Z}}_{\rho}^{o}\right)\right. \\
\left.\quad+\tilde{D}_{2}\left(-\tilde{K}_{12} \tilde{\xi}_{1}^{T} \tilde{\mathbf{Z}}_{\rho}^{o}+\tilde{T}_{1} \tilde{\xi}_{2}^{T} \tilde{\mathbf{Z}}_{\rho}^{o}\right)\right],
\end{aligned}
$$

where $D_{c c}$ denotes

$$
D_{c c}=\operatorname{det}\left(\tan \tilde{\beta}+\tilde{K}^{c c}\right) .
$$

Let us introduce the line profile indices, which are the generalization of $\tilde{q}_{1}$ and $\tilde{q}_{2}$ defined as $-\tilde{D}_{1} / \tilde{D}^{o} \tilde{\xi}_{1}$ and $-\tilde{D}_{2} / \tilde{D}^{o} \tilde{\xi}_{2}$, respectively, in a 3 channel QDT as follows:

$$
\begin{aligned}
& \tilde{q}_{1 \rho}=-\frac{\tilde{D}_{1}}{\tilde{d}_{\rho}^{(e)}} \frac{\tilde{\xi}_{1}^{T} \tilde{\mathbf{Z}}_{\rho}^{o}}{\tilde{\xi}_{1}^{T} \tilde{\xi}_{1}}=-\frac{\tilde{D}_{1}}{\tilde{d}_{\rho}^{(e)}} \frac{\tilde{\xi}_{1 \rho}^{(e)}}{\tilde{W}_{1}}, \\
& \tilde{q}_{2 \rho}=-\frac{\tilde{D}_{2}}{\tilde{d}_{\rho}^{(e)}} \frac{\tilde{\xi}_{2}^{T} \tilde{\mathbf{Z}}_{\rho}^{o}}{\tilde{\xi}_{2}^{T} \tilde{\xi}_{2}}=-\frac{\tilde{D}_{2}}{\tilde{d}_{\rho}^{(e)}} \frac{\tilde{\xi}_{2 \rho}^{(e)}}{\tilde{W}_{2}},
\end{aligned}
$$

where $\tilde{\xi}_{i}^{T} \tilde{\mathbf{Z}}_{\rho}^{o}$ is identified with the coupling matrix $\tilde{\xi}_{i \rho}^{(e)}$ between the closed channel $i$ and the eigenchannel $\rho$. The vector length $\tilde{\xi}_{i}$ of $\tilde{\xi}_{i}$ is invariant of the representation, i.e. $\tilde{\xi}_{i}^{T} \tilde{\xi}_{i}=\tilde{\xi}_{i}^{(e) T} \tilde{\xi}_{i}^{(e)}=$ $\tilde{W}_{i}(i=1,2)$ under an orthogonal transformation $Z_{i \rho}^{o}$. This suggests that there is no need to specify the representation for $\tilde{\xi}_{i}$ and $\tilde{W}_{i}$. The generalized profile indices $\tilde{q}_{1 \rho}$ and $\tilde{q}_{2 \rho}$ are not energy independent because the eigenchannels themselves change as functions of energy. The following can be obtained by substituting Eqs. (17) and (18) into Eq. (15) and after further transformations:

$$
\begin{aligned}
& \tilde{D}_{\rho}^{(e)}=\tilde{d}_{\rho}^{(e)} \tilde{W}_{2}\left(\tilde{\varepsilon}_{2}+\tilde{q}_{2 \rho}\right) \tilde{W}_{1} \\
& \times\left[\tilde{\varepsilon}_{1}+\tilde{q}_{1 \rho}-\frac{\left(\tilde{q}_{W} \cos \theta-\tilde{q}_{1 \rho} r_{\rho}\right)\left(\tilde{q}_{W} \cos \theta-\tilde{q}_{2 \rho} / r_{\rho}\right)}{\tilde{\varepsilon}_{2}+\tilde{q}_{2 \rho}}\right] \\
& \times \frac{\cos \tilde{\delta}_{\rho}}{D_{c c}}
\end{aligned}
$$

where another new parameter $r_{\rho}$ is introduced,

$$
r_{\rho}=\frac{\left(\tilde{\xi}_{2} / \tilde{\xi}_{2}\right) \cdot \tilde{\mathbf{Z}}_{\rho}^{o}}{\left(\tilde{\xi}_{1} / \tilde{\xi}_{1}\right) \cdot \tilde{\mathbf{Z}}_{\rho}^{o}}=\frac{\tilde{\xi}_{2 \rho}^{(e)} / \tilde{\xi}_{2}^{(e)}}{\tilde{\xi}_{1 \rho}^{(e)} / \tilde{\xi}_{1}^{(e)}},
$$


which represents the eigenchannel dependency of the normalized ratio of the coupling strengths. Note that the modulus of the coupling vector is independent of the representations so that $\tilde{\xi}_{i}^{(e)}=\tilde{\xi}_{i}$ [The parameter $\tilde{q}_{W}$ defined in Eq. (13) can also be represented as $\left.-\tilde{K}_{12} /\left(\tilde{\xi}_{1}^{(e) T} \tilde{\xi}_{2}^{(e)}\right)\right]$. In terms of effective reduced energy $\tilde{\varepsilon}_{\text {leff }}$ defined in Eq. (12), Eq. (21) can be rewritten in the following form:

$$
\tilde{D}_{\rho}^{(e)}=\tilde{d}_{\rho}^{(e)} \tilde{W}_{2}\left(\tilde{\varepsilon}_{2}+\tilde{q}_{2 \rho}\right) \tilde{W}_{1 \text { eff }}\left(\tilde{\varepsilon}_{1 e f f}+\tilde{q}_{1 \rho e f f}\right) \frac{\cos \tilde{\delta}_{\rho}}{D_{c c}},
$$

where

$$
\begin{aligned}
\tilde{q}_{1 \rho e f f}= & \tilde{S}+\frac{\tilde{W}_{1}}{\tilde{W}_{1 e f f}} \\
& \times\left[\tilde{q}_{1 \rho}-\frac{\left(\tilde{q}_{W} \cos \theta-\tilde{q}_{1 \rho} r_{\rho}\right)\left(\tilde{q}_{W} \cos \theta-\hat{q} / r_{\rho}\right)}{\tilde{\varepsilon}_{2}+\tilde{q}_{2 \rho}}\right]
\end{aligned}
$$

with a shift parameter $\tilde{S}$ in resonance position

$$
\tilde{S}=\frac{\tilde{W}_{1}}{\tilde{W}_{\text {leff }}}\left[\cos ^{2} \theta \frac{\tilde{\varepsilon}_{2}\left(\tilde{q}_{W}^{2}-1\right)-2 \tilde{q}_{W}}{\tilde{\varepsilon}_{2}^{2}+1}\right] \text {. }
$$

Note that $\tilde{\varepsilon}_{\text {leff }}=\left(\tilde{W}_{1} / \tilde{W}_{\text {leff }}\right) \tilde{\varepsilon}_{1}-\tilde{S}$ holds with $\tilde{S}$ defined in Eq. (25).

In order to formulate Eq. (23) into Fano-Beutler form, the last term on the right side should be related to the modulus $C$ of $\operatorname{det}\left(\tan \tilde{\beta}+\tilde{\kappa}^{c c}\right)$ defined in (10), as follows:

$$
\begin{aligned}
\frac{\cos \tilde{\delta}_{\rho}}{D_{c c}} & =\frac{\cos \tilde{\delta}_{r}}{D_{c c}} \frac{\cos \tilde{\delta}_{\rho}}{\cos \tilde{\delta}_{r}} \\
& =\frac{1-\sin ^{2} \theta\left(\tilde{W}_{1} \tilde{W}_{2} / D_{c c}\right)}{C} \frac{\cos \tilde{\delta}_{\rho}}{\cos \tilde{\delta}_{r}},
\end{aligned}
$$

where $C \cos \tilde{\delta}_{r}=D_{c c}-\tilde{W}_{1} \tilde{W}_{2} \sin ^{2} \theta$ is used. The total photoionization cross section can be expressed as follows by substituting Eqs. (10) and (26) into (23):

$$
\begin{aligned}
\sigma & =\frac{4 \pi^{2} \alpha \omega}{3} \sum_{\rho}\left|\tilde{D}_{\rho}\right|^{2} \\
& =\frac{4 \pi^{2} \alpha \omega}{3} \sum_{\rho} \tilde{d}_{\rho}^{(e) 2} \frac{\left(\tilde{\varepsilon}_{2}+\tilde{q}_{2 \rho}\right)^{2}}{\tilde{\varepsilon}_{2}^{2}+1} \frac{\left(\tilde{\varepsilon}_{1 e f f}+\tilde{q}_{1 \rho e f f}\right)^{2}}{\tilde{\varepsilon}_{1 e f f}^{2}+1} A_{\rho}^{2},
\end{aligned}
$$

where $\omega$ is the wave-number of the absorbed photon, $\alpha$ the hyperfine constant and $A_{\rho}$ denotes

$$
A_{\rho}=\left(1-\frac{\tilde{W}_{1} \tilde{W}_{2}}{D_{c c}} \sin ^{2} \theta\right) \frac{\cos \tilde{\delta}_{\rho}}{\cos \tilde{\delta}_{r}} .
$$

This shows the extent of the avoided crossing interactions and is close to unity in most ranges of energy except for approximately the singular or kink points.

In Eq. (27), the effective parameters of $\tilde{\varepsilon}_{\text {leff }}$ and $\tilde{q}_{1 \rho e f f}$ reflect the perturbation of the dense Rydberg series by an interloper. If there are no perturbations, they would simply be given by $\tilde{\varepsilon}_{1}$ and $\tilde{q}_{1 \rho}$ with $\tilde{q}_{1 \rho}$ as a constant. The poles in $\tilde{q}_{1 \rho e f f}$ indicate huge intensity enhancement of the spectrum of the dense Rydberg series due to the perturbation of an interloper. Since there is no overall change in the interloper spectrum, the enhancement should come from the other part of its own spectrum, mostly from the zero part of $\tilde{q}_{1 \rho e f f}$.

The validity of formulation (27) can be checked by comparing the numerical results with the ones obtained from

$$
\begin{aligned}
\sigma & =\frac{4 \pi^{2} \alpha \omega}{3} \sum_{\rho}\left|\tilde{D}_{\rho}\right|^{2} \\
& =\frac{4 \pi^{2} \alpha \omega}{3} \tilde{\mathbf{D}}^{o}\left(1+\tilde{\mathbf{K}}^{2}\right)^{-1} \tilde{\mathbf{D}}^{o}
\end{aligned}
$$

where $\tilde{\mathbf{D}}^{o}$ denotes $\tilde{D}^{o}-\tilde{D}^{c}\left(\tan \tilde{\beta}+\tilde{K}^{c c}\right)^{-1} \tilde{K}^{c o}$, which is representation in the standing wave channel basis functions $\tilde{\Psi}_{i}$. $\tilde{\Psi}_{i}$ is not energy normalized and requires the normalization factor $\left(1+\tilde{\mathbf{K}}^{2}\right)^{-1}$ included in Eq. (29).

The envelope of the spectra is served by the spectrum of an interloper, which is given by

$$
\sigma^{I}=\sum_{\rho} \sigma_{\rho}^{I}=\frac{4 \pi^{2} \alpha \omega}{3} \sum_{\rho} \tilde{d}_{\rho}^{(e) 2} \frac{\left(\tilde{\varepsilon}_{2}+\tilde{q}_{2 \rho}\right)^{2}}{\tilde{\varepsilon}_{2}^{2}+1}
$$

The envelope $\sigma_{\max }$ that connects the maxima of the cross section spectrum is obtained by replacing the perturbed spectrum $\left(\tilde{\varepsilon}_{1 e f f}+\tilde{q}_{1 \rho e f f}\right)^{2} /\left(\tilde{\varepsilon}_{1 \text { eff }}^{2}+1\right)$ of the dense Rydberg series by its maximum $1+\tilde{q}_{1 \rho e f f}^{2}$ and setting $A_{\rho}$ to unity, which amounts to ignoring the complexity caused by the avoided crossing interactions, leaving only resonant interactions:

$$
\begin{aligned}
\sigma_{\max } & =\frac{4 \pi^{2} \alpha \omega}{3} \sum_{\rho} \tilde{d}_{\rho}^{(e) 2} \frac{\left(\tilde{\varepsilon}_{2}+\tilde{q}_{2 \rho}\right)^{2}}{\tilde{\varepsilon}_{2}^{2}+1}\left(1+\tilde{q}_{1 \rho e f f}^{2}\right) \\
& =\sum_{\rho} \sigma_{\rho}^{I}\left(1+\tilde{q}_{1 \rho e f f}^{2}\right)
\end{aligned}
$$

$1+\tilde{q}_{1 \rho e f f}^{2}$ acts as an enhancement factor to the interloper's spectrum from the interloper's viewpoint. The behavior of an enhancement factor $1+\tilde{q}_{1 \rho e f f}^{2}$ is greatly affected by the poles and zeros of $\tilde{q}_{1 \rho e f f}$. The next section considers the poles and zeros of $\tilde{q}_{1 \rho \text { eff }}$.

Poles and zeros of $\widetilde{\boldsymbol{q}}_{1 \rho e f f}$. In previous work, ${ }^{3}$ the pole and zero structures play an important role in determining the shape of the complex spectra caused by overlapping resonances in the system involving 1 open and 2 closed channels. Let us see how the pole and zero structures change when more than one open channel is involved. Note that singular points in $\tilde{\varepsilon}_{i}$ are irrelevant to the discussion. This study was interested in the induced singular points or poles in the effective parameters, $\tilde{\varepsilon}_{1 \text { eff }}$ and $\tilde{q}_{1 \rho \text { eff }}$, which 
describe the perturbed spectra of the dense Rydberg series through channel coupling with an interloper due to overlapping resonances. The resonance structures in the perturbed spectra of the dense Rydberg series are related to the poles of $\tilde{\varepsilon}_{1 \text { eff }}$, and the spectral shapes are represented by the asymmetric line profile indices $\tilde{q}_{1 \rho e f f}$. Since $\tilde{\varepsilon}_{1 \text { leff }}$ involves only the channel coupling strengths, it has a simpler pole structure than that in $\tilde{q}_{1 \rho e f f}$. Let us first examine the pole structure of $\tilde{\varepsilon}_{\text {leff }}$. Substitution of Eq. (11) into (12) and decomposition into partial fractions yield

$$
\begin{aligned}
\tilde{\varepsilon}_{1 e f f}= & \tilde{\varepsilon}_{1}-\cos ^{2} \theta \\
& \times \frac{\left(\tilde{\varepsilon}_{2}+\tilde{q}_{W}\right)\left(2 \tilde{q}_{W} \tilde{\varepsilon}_{1}+\tilde{q}_{W}^{2}-1\right)-\left(\tilde{\varepsilon}_{1}+\tilde{q}_{W}\right)\left(\tilde{q}_{W}^{2}+1\right)}{\left(\tilde{\varepsilon}_{2}+\tilde{q}_{W}\right)^{2} \cos ^{2} \theta+\left(\tilde{\varepsilon}_{2}^{2}+1\right) \sin ^{2} \theta}
\end{aligned}
$$

This shows that the second order pole at $\tilde{\varepsilon}_{2}=-\tilde{q}_{W}$ observed in the 3 channel QDT disappears due to the second term $\left(\tilde{\varepsilon}_{2}^{2}+1\right) \sin ^{2} \theta$, which enters when there is incomplete superimposition of the overlapping resonances so that $\theta \neq 0$ or $\pi$. In the 3 channel QDT, $\theta$ is always either 0 or $\pi$ and the resonances are always superimposed. Therefore, as far as the overlapping resonances are partially superimposed, there is no pole at $\tilde{\varepsilon}_{2}=-\tilde{q}_{W}$. Eq. (32) shows that $\tilde{\varepsilon}_{1 \text { eff }}$ still has a singular point at $\tilde{\varepsilon}_{1} \rightarrow \infty$, which occurs at every spectral interval of the Rydberg series (note that the singular point of $\tilde{\varepsilon}_{2} \rightarrow \infty$ cancels out), indicating that the involvement of more open channels does not alter the periodic nature of the spectrum of the Rydberg series.

Similarly, one can decompose $\tilde{q}_{1 \rho e f f}$ into partial fractions as follows:

$$
\begin{aligned}
\tilde{q}_{1 \rho e f f}= & \tilde{q}_{1 \rho}+\frac{A}{\tilde{\varepsilon}_{2}+\tilde{q}_{2 \rho}} \\
& +B \frac{C \tilde{\varepsilon}_{2}+D}{\left(\tilde{\varepsilon}_{2}+\tilde{q}_{W} \cos ^{2} \theta\right)^{2}+\sin ^{2} \theta\left(1+\tilde{q}_{W}^{2} \cos ^{2} \theta\right)}
\end{aligned}
$$

where the explicit forms of $A, B, C$ and $D$ have no utility and are ignored. If $A, B, C$ and $D$ have constant energy or are insensitive to energy variations, Eq. (33) suggests that $\tilde{q}_{1 \rho e f f}$ will have a simple pole at $-\tilde{q}_{2 \rho}$. In rare cases, it would have a second order pole at $\tilde{\varepsilon}_{2}=-\tilde{q}_{W}$ if $\theta=0$, which is in contrast to the 3 channel QDT, where the pole at $\tilde{\varepsilon}_{2}=-\tilde{q}_{W}$ is a simple pole. Note that $\tilde{q}_{1 \rho}$ and $\tilde{q}_{2 \rho}$ do not have constant energy or are at least insensitive to energy but are generally functions of energy.

The simple pole at $\tilde{\varepsilon}_{2}=-\tilde{q}_{W}$ observed in systems involving 1 open and 2 closed channels is no longer a pole in the systems involving more than 1 open channel if $\theta \neq 0$. If overlapping resonances are completely super-imposable, i.e., $\theta=0$, there is no $q$-reversal across $\tilde{\varepsilon}_{2}=-\tilde{q}_{W}$ because the pole is of second order. However, the enhancement still remains, even though the range of enhancement is much narrower due to the nature of the second pole. However, if $\theta \neq 0$, there is no $q$ reversal and no enhancement around $\tilde{\varepsilon}_{2}=-\tilde{q}_{W}$. The direct closed channel coupling $\tilde{K}_{12}$ acts on the spectra only via $\tilde{q}_{W}$ and its effect on the spectra becomes less conspicuous if more than one open channel is involved. The zero surface $\tilde{q}_{1 \rho \text { eff }}\left(\tilde{\varepsilon}_{2}\right)=0$ obtained from (33) is quite complicated in general.

The case of $\tilde{\mathbf{D}}^{o}=$ zero in a 4 channel system with 2 open channels. If $\tilde{\mathbf{D}}^{o}=\left(\tilde{D}_{3}, \tilde{D}_{4}\right)$ are the zero vector, $\tilde{d}_{\rho}^{(e)}$ is zero and the asymmetric line profile parameters $\tilde{q}_{i \rho}(i=1,2)$ become singular and indefinable. However, $\tilde{d}_{\rho}^{(e)} \tilde{q}_{i \rho}$ is finite. If it is denoted as $\tilde{p}_{i \rho}$, it is given by $-\tilde{D}_{i} \tilde{\xi}_{i \rho}^{(e)} / \tilde{W}_{i}$, and $p_{2 \rho} / p_{1 \rho}$ can be related to $r_{\rho}$ as $p_{2 \rho} / p_{1 \rho}=r_{\rho} / r$ with the introduction of another parameter $r$ :

$$
r=\frac{\tilde{D}_{1} \tilde{\xi}_{2}}{\tilde{D}_{2} \tilde{\xi}_{1}}
$$

In this case, $\tilde{q}_{1 \rho e f f}$ becomes

$$
\begin{aligned}
& \tilde{q}_{1 \rho e f f} \\
& =\frac{\cos ^{2} \theta\left[\tilde{\varepsilon}_{2}\left(\tilde{q}_{W}^{2}-1\right)-2 \tilde{q}_{W}\right]+\frac{r}{r_{\rho}}\left(\tilde{\varepsilon}_{2}+t_{\rho}\right)\left(\tilde{\varepsilon}_{2}^{2}+1\right)}{\left(\tilde{\varepsilon}_{2}+\tilde{q}_{W}\right)^{2} \cos ^{2} \theta+\left(\tilde{\varepsilon}_{2}^{2}+1\right) \sin ^{2} \theta},
\end{aligned}
$$

where $t_{\rho}$ denotes

$$
t_{\rho}=\tilde{q}_{W} \cos \theta\left(r^{-1}+r_{\rho}\right) .
$$

Eq. (35) shows that $\tilde{q}_{1 \rho e f f}$ does not have a pole at $\tilde{\varepsilon}_{2}=-\tilde{q}_{2 \rho}$ because $\tilde{q}_{2 \rho}$ approaches infinity when $\tilde{d}_{\rho}^{(e)} \rightarrow 0$ and cannot be defined in this case.

Another key step in the use of a general cross section formula (27) lies in the identification of one of the eigenchannels as a resonant one and the other as a non-resonant one, as recognized by Cohen. ${ }^{8}$ To expound this point, let us consider the solution of Eq. (6) in the present system. For the system involving 2 open channels, the compatibility equation (6) is the quadratic equation given by $\tan ^{2} \tilde{\delta}_{\rho}-\operatorname{tr}(\tilde{\mathbf{K}}) \tan \tilde{\delta}_{\rho}+\operatorname{det}(\tilde{\mathbf{K}})=0$. For convenience, let us adopt Cohen's notation $D_{c c} \tan ^{2} \tilde{\delta}_{\rho}+B \tan \tilde{\delta}_{\rho}+C=0$ for the quadratic equation where $B=-D_{c c} \operatorname{tr}(\tilde{\mathbf{K}})$ and $C=D_{c c}$ $\operatorname{det}(\tilde{\mathbf{K}})$, or

$$
\begin{aligned}
& B=\left(\tilde{K}_{23}^{2}+\tilde{K}_{24}^{2}\right) \tilde{T}_{1}+\left(\tilde{K}_{13}^{2}+\tilde{K}_{14}^{2}\right) \tilde{T}_{2}-2 \tilde{K}_{12} \tilde{\xi}_{1} \cdot \tilde{\xi}_{2} \\
& C=\left(\tilde{\xi}_{1} \times \tilde{\xi}_{2}\right)^{2}=\left(\tilde{K}_{13} \tilde{K}_{24}-\tilde{K}_{14} \tilde{K}_{23}\right)^{2} .
\end{aligned}
$$

Here, $\tilde{T}_{i}$ denotes $\tan \tilde{\beta}_{i}$. Cohen reported that the unequal partitioning of the resonant features occurs if the solution is chosen to be the most trouble-free form:

$$
\begin{aligned}
& \tan \tilde{\delta}_{r e s}=-\frac{A}{D_{c c}} \\
& \tan \tilde{\delta}_{n r}=-\frac{C}{A},
\end{aligned}
$$


where $A$ denotes

$$
A=\frac{1}{2}\left[B+\operatorname{sign}(B) \sqrt{B^{2}-4 D_{c c} C}\right] .
$$

A numerical study reveals that $r_{\rho}$ is almost constant of energy except in the neighborhood of the kink located at $B=0$, which corresponds to $\operatorname{tr}(\tilde{\mathbf{K}})=0$. Note that the point of kink is not a singular point and, e.g. $q$ reversal does not happen at this point because the sign function in Eq. (39) ensures no sign change. Therefore, the only effect the point of the kink has on the spectra is the enhancement. Its presence complicates the curve equation of $\tilde{q}_{1 \rho e f f}$ and it is difficult to find its root. The removal of a kink will greatly simplify the analysis without losing its validity because kinks are not associated with $q$ reversals. One way of removing the kink is to use the averaged value of $r_{\rho}$ for $\rho=r e s$ or $r_{\text {res }}$. The other simple way is to calculate $r_{\text {res }}$ at the most remote point from the kink. $\tilde{\varepsilon}_{1}=\infty$ or $\tan \tilde{\beta}_{1}=\tilde{T}_{1}=\infty$ corresponds to such a point. Fortunately, it is easy to obtain an analytic solution at this point due to the dominance of the term containing $\tilde{T}_{1} \cdot \tan \tilde{\delta}_{\text {res }}$ takes the value of $-\tilde{W}_{2} \tilde{T}_{1} / D_{c c}$ at this point, which yields the solution $\tilde{\mathbf{Z}}_{o}^{\text {res }} \equiv \tilde{\mathbf{Z}}_{\rho=\text { res }}^{o}=\left(\tilde{Z}_{3}^{\text {res }}, \tilde{Z}_{4}^{\text {res }}\right)$ of Eq. (7) as

$$
\tilde{Z}_{4}^{r e s} / \tilde{Z}_{3}^{r e s}=\tilde{K}_{24} / \tilde{K}_{23}
$$

Substituting this into (22), one obtains $r_{\text {res }}=\cos ^{-1} \theta$, where $t_{\text {res }}$ defined in Eq. (36) and becomes $\tilde{q}_{W}\left(1-r^{-1} \cos \theta\right)$. From this, one obtains

$$
\begin{aligned}
& \tilde{q}_{1 \text { eff }}^{\text {res }} \equiv \tilde{q}_{1 \rho=\text { res eff }} \\
& =r \cos \theta \frac{\tilde{\varepsilon}_{2}^{3}+\tilde{q}_{W}(1+t) \tilde{\varepsilon}_{2}^{2}+\left\lfloor 1+t\left(\tilde{q}_{W}^{2}-1\right)\right\rfloor \tilde{\varepsilon}_{2}+\tilde{q}_{W}(1-t)}{\left(\tilde{\varepsilon}_{2}+\tilde{q}_{W}\right)^{2} \cos ^{2} \theta+\left(\tilde{\varepsilon}_{2}^{2}+1\right) \sin ^{2} \theta}
\end{aligned}
$$

where $t$ denotes $\cos \theta / r$.

Zero surfaces of $\tilde{q}_{\text {leff }}^{\text {res }} q$ reversals occur either at simple poles or at zeros of $\tilde{q}_{1 \text { eff }}^{r e s}$. Eq. (41) suggests that $\tilde{q}_{\text {leff }}^{r e s}$ has no simple pole if overlapping resonances are not completely super-imposable so that $\theta \neq 0$. However, $\tilde{q}_{1 e f f}^{\text {res }}\left(\tilde{\varepsilon}_{2}\right)=0$ can yield as many as 3 zero points of $\tilde{\varepsilon}_{2}$ corresponding to the real roots of the cubic equation obtained from its numerator:

$$
\tilde{\varepsilon}_{2}^{3}+\tilde{q}_{W}(1+t) \tilde{\varepsilon}_{2}^{2}+\left[1+t\left(\tilde{q}_{W}^{2}-1\right)\right] \tilde{\varepsilon}_{2}+\tilde{q}_{W}(1-t)=0 .
$$

Since the cubic equation contains two parameters, $\tilde{q}_{W}$ and $t$, the roots form zero surfaces in the space of $\tilde{q}_{W}$ and $t$. How many zero surfaces there are can be determined from the sign of the discriminant $\Delta$ of the cubic equation which are given by $18 a b c$ $4 a^{3} c+a^{2} b^{2}-4 b^{3}-27 c^{2}$ for the cubic equation $x^{3}+a x^{2}+b x+c$. If it is positive, the equation has 3 distinct real roots. If it is zero, the equation has a multiple root and all its roots are real. If it is negative, the equation has only one real root. The discriminant of Eq. (42) can be obtained as a surprisingly simple form:

$$
\Delta=\left(\tilde{q}_{W}^{2}+1\right)^{2}\left[t^{2} \tilde{q}_{W}^{2}+4(t-1)\right](t-1)^{2} .
$$

It tells us that if $t>1, \Delta$ is always positive indicating 3 distinct zero surfaces. If $t=1, \Delta=0$ and two of the zero surfaces meet on a line. If $t<1, \Delta$ is negative in the inside region enclosed by the curves $t=-2\left(1 \pm \sqrt{1+\tilde{q}_{W}^{2}}\right) / \tilde{q}_{W}^{2}$ and positive outside the region as shown in Fig. 1. Although it is difficult to see in Fig. 1, a careful observation shows that three surfaces first transform to one surface via the meeting of two of them on a line as a multiple root and then disappears leaving one behind. The number of zero surfaces can be one and three depending on the parameter ranges.

The case of $\tilde{K}_{12}$ equal to zero in 4 channel system with 2 open channels. Let us consider the case in which channel coupling between two closed channels is zero, as observed ${ }^{10}$ in the photoionization spectra of the Rydberg series $3 d^{9} 4 s\left({ }^{1} D_{2}\right) n d^{2} G_{9 / 2}$ $(n=16-20)$ perturbed by the interloper $3 d^{9} 4 p^{2}{ }^{4} F_{9 / 2}$. In this case, $\tilde{q}_{W}=0$ and subsequently, $t_{\rho}=0$. The spectral width of the perturbed Rydberg series can be simplified by substituting these into Eq. (35) as follows:

$$
\tilde{W}_{1 e f f}=\tilde{W}_{1} \frac{\tilde{\varepsilon}_{2}^{2}+\sin ^{2} \theta}{\tilde{\varepsilon}_{2}^{2}+1}
$$

and $\tilde{q}_{1 \rho e f f}$ can be obtained regardless of the type of $\rho$ as follows:

$$
\tilde{q}_{1 \rho e f f}=\frac{\tilde{\varepsilon}_{2}}{\tilde{\varepsilon}_{2}^{2}+\sin ^{2} \theta} \frac{\tilde{\varepsilon}_{2}^{2} \tilde{q}_{1 \rho}-\tilde{\varepsilon}_{2} \cos ^{2} \theta+\tilde{q}_{1 \rho}-\tilde{q}_{2 \rho} \cos ^{2} \theta}{\tilde{\varepsilon}_{2}+\tilde{q}_{2 \rho}}
$$

This shows that $\tilde{q}_{1 \rho \text { eff }}$ has a simple pole at $\tilde{\varepsilon}_{2}=-\tilde{q}_{2 \rho}$ and has at least one at $\tilde{\varepsilon}_{2}=0$, and the other zeros are determined by the nature of the quadratic equation $\tilde{\varepsilon}_{2}^{2} \tilde{q}_{1 \rho}-\tilde{\varepsilon}_{2} \cos ^{2} \theta+\tilde{q}_{1 \rho}-\tilde{q}_{2 \rho}$ $\cos ^{2} \theta=0$.

Application of the theory to the autoionizing Rydberg series Cu I 3 $\boldsymbol{d}^{\mathbf{9}}$ 4snl. Martins and Zimmermann ${ }^{10}$ obtained unusual photoionization spectra of the Rydberg series $\mathrm{Cu} \mathrm{I} 3 d^{9} 4 s\left({ }^{1} D_{2}\right)$ nd

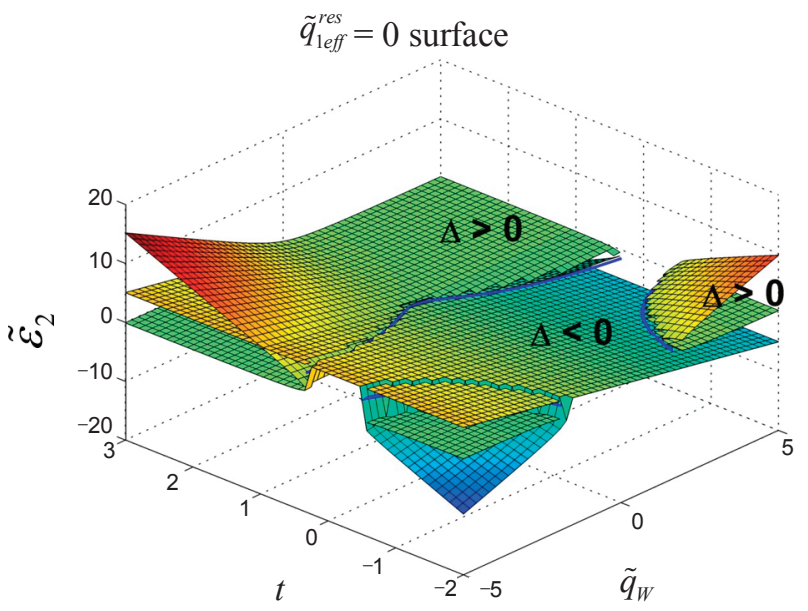

Figure 1. $\tilde{q}_{\text {leff }}^{\text {res }}=0$ surfaces. See Eqs. (42) and (43) for details. 
${ }^{2} G_{9 / 2}\left(n=16\right.$ - 20) perturbed by the interloper $3 d^{9} 4 p^{2}{ }^{4} F_{9 / 2}$, and explained the spectrum using a 4 channel QDT with the two open channels, $3 d^{9} 4 s\left({ }^{3} D_{3}\right) \varepsilon d^{2} G_{9 / 2}$ and $3 d^{9} 4 s\left({ }^{3} D_{2}\right) \varepsilon d^{4} G_{9 / 2}$. They also obtained a 3 channel version of the spectrum, which appeared completely different and showed numerically how a different 3 channel QDT spectrum transforms to the observed spectrum by turning the interactions of 2 closed channels with the newly included open channel on progressively. Cohen applied his 4 channel QDT to this system and found that his theory failed for this system because the direct interaction $\tilde{K}_{12}$ between the closed channels is negligible compared with the indirect interaction $\tilde{K}_{13} \tilde{K}_{23}$ via open channel 3 to which his theory cannot be applied.

However, this is the system belonging to the described cases for which our theory was fully developed. Therefore, in contrast to his theory, this system provides a meaningful scheme to which the present theory can be applied. This possibility is very important because with this system we can trace how the additional open channel affects the channel coupling whereby a completely different 3 channel spectrum evolves into the strange 4 channel spectrum. On the other hand, more fundamentally, this system shows how the perturbation of the autoionization spectra of the dense Rydberg series is affected by an additional open channel.

The theory was applied first to the model study conceived by Martins and Zimmermann to emulate the observed spectra from a 3 channel spectrum by turning the channel coupling with an additional open channel on. The theory was then applied to the experimental spectra.

Systematic study of the spectral changes due to an additional open channel. Let us apply the theory to the model system chosen by Martins and Zimmermann with the same parameters except for the value of the transition dipole moment $\tilde{D}_{3}$, the value of which was taken as 0.5 by them but was set to zero in this study without altering the spectra significantly. The values of the other parameters are denoted in the figure caption. First consider the system involving only 3 channels with channels 1 and 2 closed and channel 3 open. The $q$ reversal patterns for this 3 channel system were examined in Ref. [3]. However, if $\tilde{D}_{3}=0$, one of the simple poles at $\tilde{\varepsilon}_{2}=-\tilde{q}_{2}$ of $\tilde{q}_{\text {leff }}$ becomes singular and the method in Ref. [3] cannot be applied directly. An extension of the theory to include this case can be easily done and described in Appendix A. It shows that $\tilde{q}_{1 e f f}$ has only one simple pole at $\tilde{\varepsilon}_{2}=-\tilde{q}_{W}$ and its zeros are obtained as roots of a different quadratic equation. Otherwise, the principle of analysis is the same as that described in Ref. [3]. Fig. 2 shows that the simple pole lies on the right side of the interloper resonance center and the huge enhancement of intensity occurs there. One of the two zeros of $\tilde{q}_{\text {eeff }}$ lies on the left side of the interloper peak and the $q$ reversal across it can be clearly discerned. Also shown is the envelope of the enhanced spectra from the interloper by $1+\tilde{q}_{\text {leff }}^{2}$ shown with the dash-dotted line. Martins and Zimmermann reported how the satisfactorily simulated spectrum evolves from a 3 channel spectrum by turning progressively the coupling strength $\tilde{K}_{24}$ between closed channel 2 with another open channel 4 on and then the coupling $\tilde{K}_{14}$ progressively on. However, their study did not explain why such changes are possible and how they evolve. Let us answer this question using the theory de-
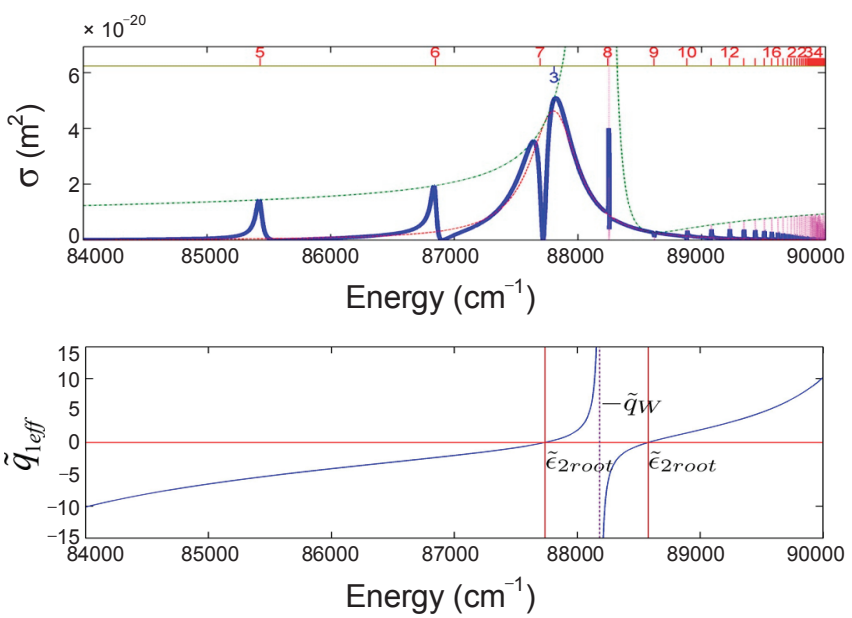

Figure 2. Photoionization cross section and $\tilde{q}_{\text {leff }}$ spectra calculated using the 3 channel QDT modeled for the $\mathrm{Cu}$ I Rydberg series $3 d^{9} 4 s\left({ }^{1} D_{2}\right) n d^{2} G_{9 / 2}$ perturbed by the interloper $3 d^{9} 4 p^{2}{ }^{4} F_{9 / 2}$. The cross section $\sigma$ is drawn with a heavy line, the envelope with a dotted line and $\sigma_{\max }$ with a dashed line. Effective quantum numbers are shown in the $\sigma$ spectrum. The upper integer values are $n_{1}^{*}$ for the dense Rydberg series and the lower one is $n_{2}^{*}$ for the interloper. This convention will be applied for all other photoionization cross sections. $\tilde{K}_{12}=0.1, \tilde{K}_{13}=0.2, \tilde{K}_{23}=0.3, \tilde{D}_{1}=3.0, \tilde{D}_{2}=9.0, \tilde{D}_{3}=0$ a.u., $I_{1}=$ $9 \times 10^{4} \mathrm{~cm}^{-1}, I_{2}=1 \times 10^{5} \mathrm{~cm}^{-1}, \tilde{\mu}_{1}=0.1, \tilde{\mu}_{2}=0.0$ a.u. are used.

\section{veloped here.}

Fig. 3 shows the photoionization cross section spectra and their $\tilde{q}_{\text {leff }}$ calculated with Eqs. (27) and (41), respectively, for the resonant eigenchannel when the channel coupling between closed channel 2 and open channel 4 is turned on. Fig. 4 shows a similar calculation for the non-resonant eigenchannel. Note that the resonant eigenchannel spectrum dominates the photoionization spectra. Its $q$ reversal pattern behaves simply, whereas many singular behaviors are observed for the nonresonant eigenchannel. This is derived from the eigenchannel design, which excludes the poles from the resonant eigenchannels from the outset by choosing the same signs for the two constituent terms so that the solution never changes sign whereby the poles are sequestered into the nonresonant eigenchannels.

Let us examine the resonant $\tilde{q}_{1 e f f}^{\text {res }}$. One of the conspicuous features is the kinks that occur at $D_{c c}=0$. Since the same sign is chosen, $\tilde{q}_{\text {leff }}^{\text {res }}$ does not change sign at the kinks located at $D_{c c}=0$. The role of kinks is only to enhance or diminish the spectra. The kinks are not important if we are interested in the $q$ reversals or sign change in $\tilde{q}_{\text {leff }}^{\text {res }}$. Previously, we showed how to remove the kinks and obtain a smoothed curve for $\tilde{q}_{\text {leff }}^{\text {res }}$. The curve is described by the cubic equations of $\tilde{\varepsilon}_{2}$. Fig. 3 shows that the cubic curve represents $\tilde{q}_{1 e f f}^{r e s}$ quite well. It also shows that it has 3 distinct real roots and causes $3 q$ reversals. Since the spectrum does not reach zero between the adjacent series lines as Rydberg series are crossed, the $q$ reversals do not cause such a large change as in the 3 channel QDT spectrum but their occurrence can still be distinguished.

Turning the channel interaction of the closed channels 1 and 2 with an additional open channel 4 along with the one with open channel 3 is performed in two schemes, first turning $\tilde{K}_{24}$ progressively on while $\tilde{K}_{14}$ is turned off. $\tilde{K}_{24}$ is then fixed to some 

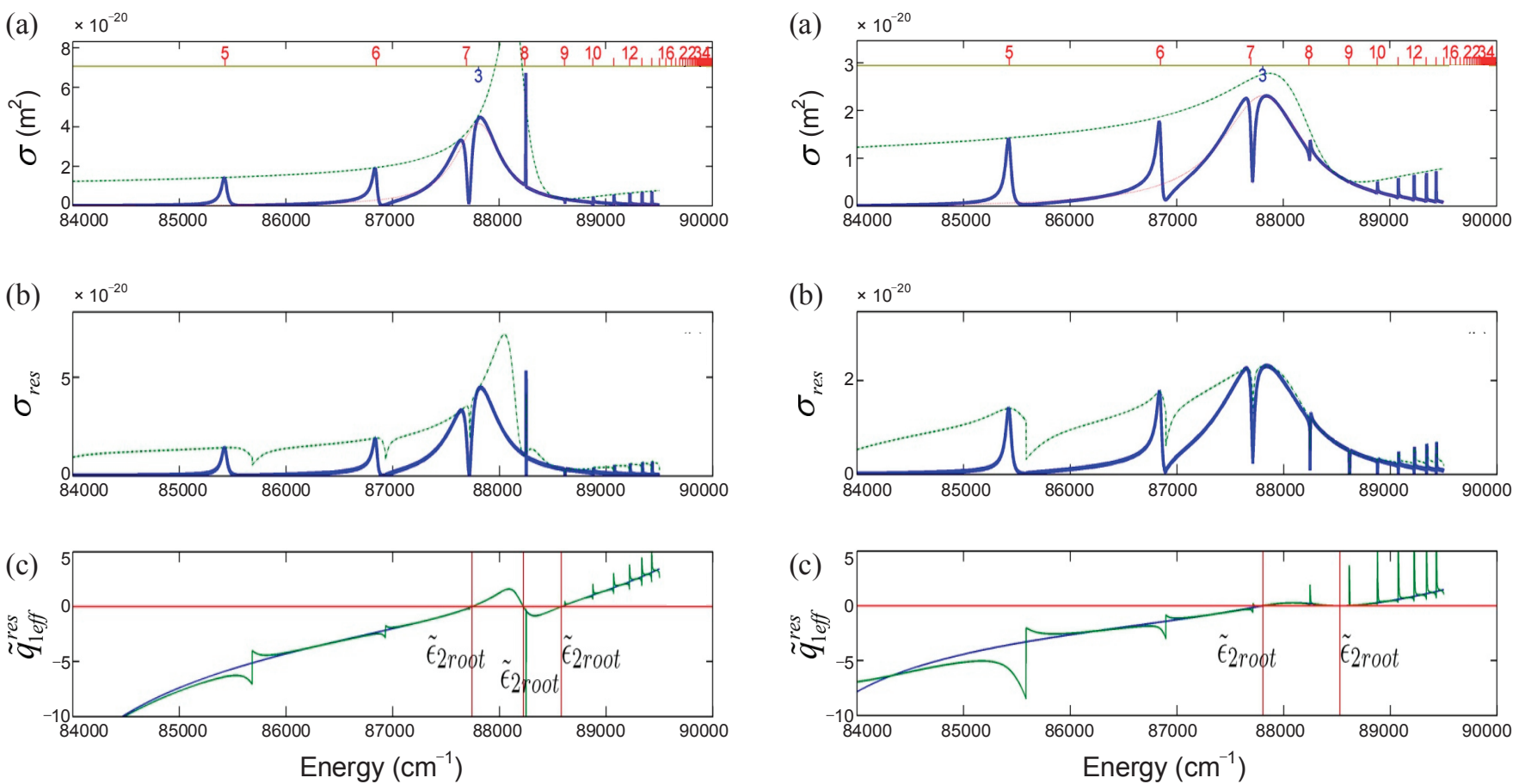

Figure 3. Photoionization cross sections and $\tilde{q}_{\text {leff }}^{\text {res }}$ calculated using the 4 channel QDT. (a) cross section $\sigma$ (b) the resonant eigenchannel component $\sigma_{\text {res }}$ (c) both $\tilde{q}_{1 e f f}^{\text {res }}$ for Eqs. (35) and (41). $\tilde{K}_{14}=0, \tilde{K}_{24}=0.1$ and $\tilde{D}_{4}=0$ a.u. are used. All other parameters used are the same as for Fig. 2. $\tilde{q}_{W}=-1.67, t=1.8$ and $\cos \theta=0.95$.

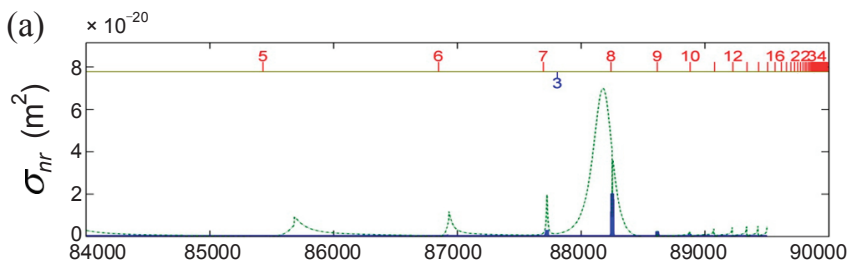

Figure 5. Similar calculations to Fig. 3 for $\tilde{K}_{24}=0.3, \tilde{q}_{W}=-1.67, t=1$ and $\cos \theta=0.71$.
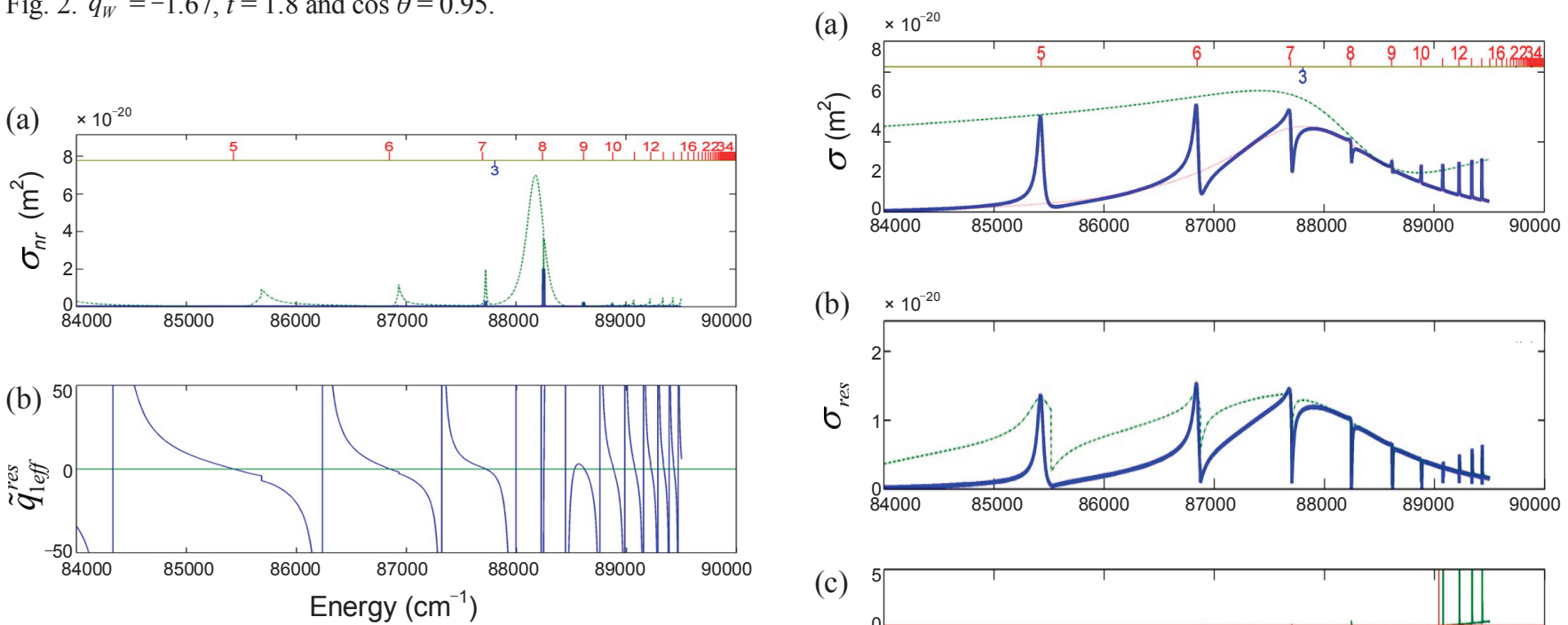

Figure 4. Corresponding calculation to the ones in Fig. 3 for the nonresonant component.

value, and $\tilde{K}_{14}$ is progressively turned on. The first scheme corresponds to a progressive decrease in $t$ while the value of $\tilde{q}_{W}$ is constant. This scheme plays the role of choosing the good starting point for the second scheme. The second scheme was designed to move the zero point of $\tilde{q}_{\text {leff }}^{\text {res }}$ to the location so that the spectrum has the same $q$ reversal pattern as the observed one. Let us uncover the underlying principle of how the scheme works by applying the current analysis tool. Dividing the turning process of the coupling of the closed channels with another open channel into two schemes is to allow easy control of the overlapping re-

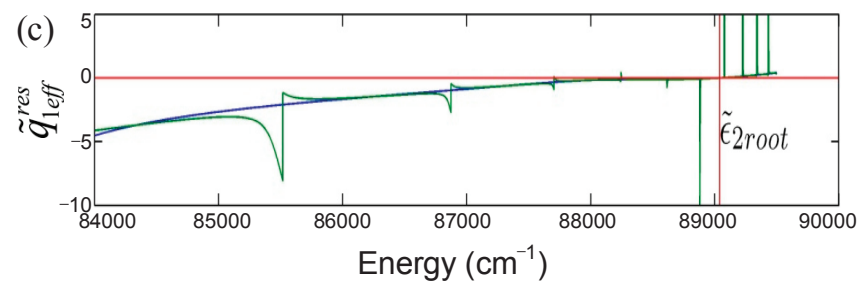

Figure 6. Similar calculations to Fig. 3 for $\tilde{K}_{24}=0.5, \tilde{q}_{W}=-1.67, t=$ 0.53 and $\cos \theta=0.51$.

sonance. Cohen's restriction to the first scheme is capable of performing the same task by varying the value of $\tilde{q}_{W}$ separately, but only with difficulty.

Consider the first scheme, where values of $\tilde{K}_{24}$ are increased progressively from 0.1 via 0.3 to 0.5 while $\tilde{K}_{14}^{24}$ is kept being 

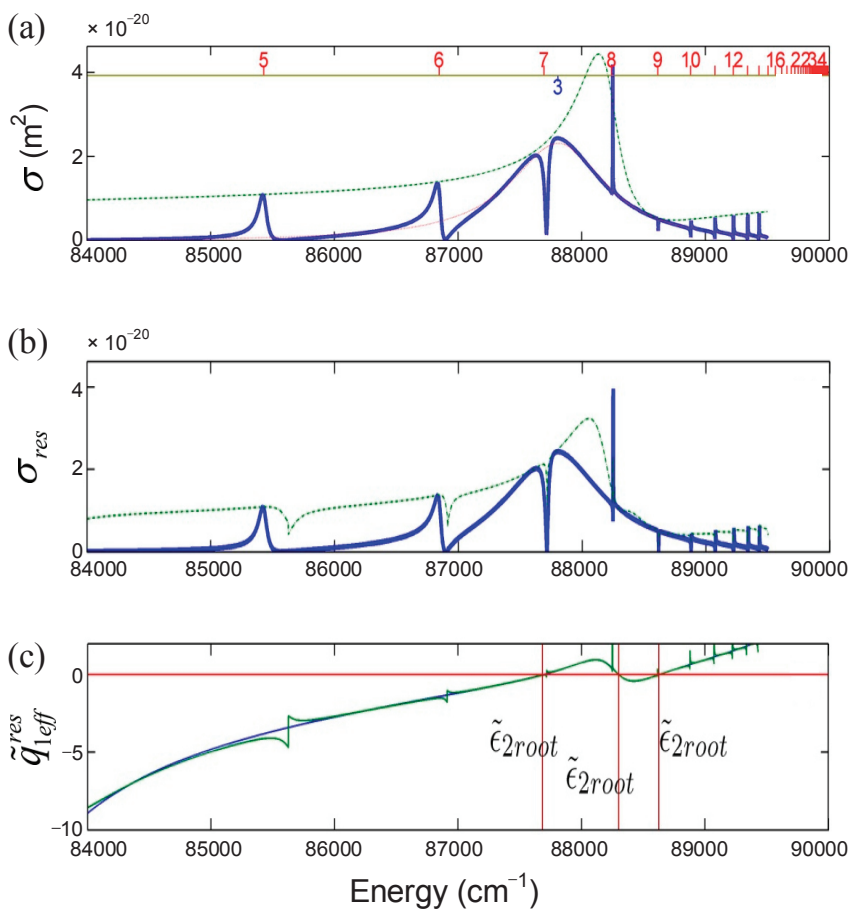

Figure 7. Similar calculations to Fig. 3 for $\tilde{K}_{24}=0.3, \tilde{K}_{14}=0.1, \tilde{q}_{W}=$ $-1.11, t=1.5$ and $\cos \theta=0.95$.
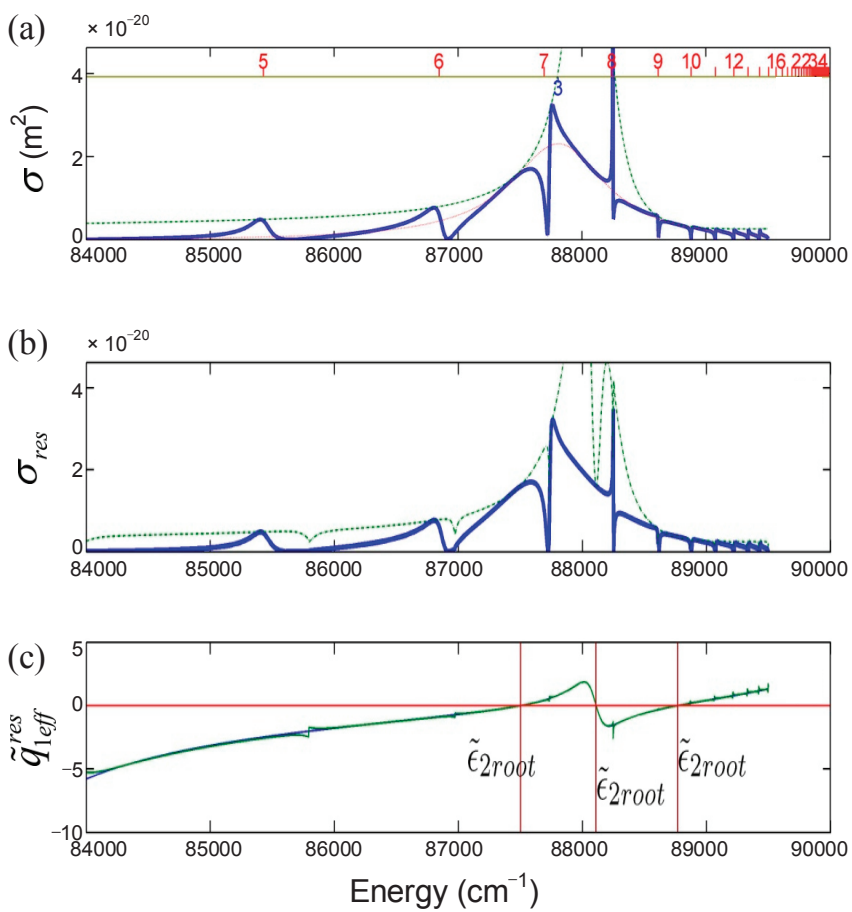

Figure 8. Similar calculations to Fig. 3 for $\tilde{K}_{24}=0.3, \tilde{K}_{14}=0.3, \tilde{q}_{W}=$ $-0.67, t=2.5$ and $\cos \theta=0.98$.

turned off. During the increase in $\tilde{K}_{24}, \tilde{q}_{W}$ remains constant while $t$ decreases from 1.80 via 1 to 0.53 . This means that initially, $\tilde{q}_{\text {leff }}^{r e s}$ has 3 distinct real roots, a multiple root and finally ends up with one real root. There are two values of $t$ correspond-
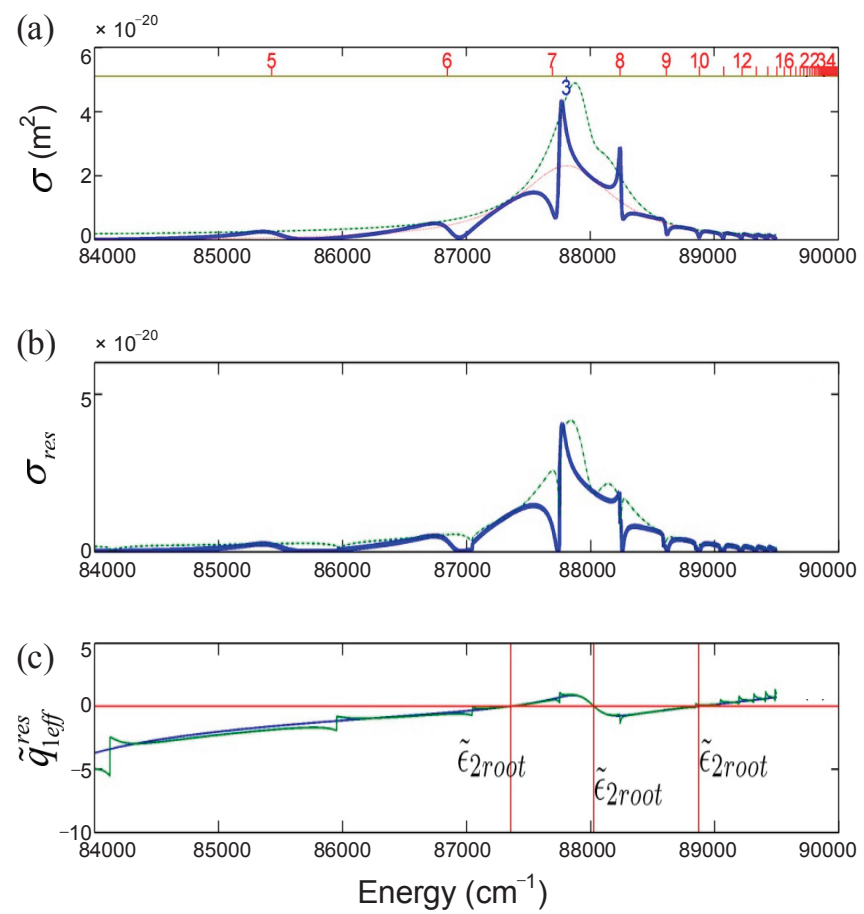

Figure 9. Similar calculations to Fig. 3 for $\tilde{K}_{24}=0.3, \tilde{K}_{14}=0.5, \tilde{q}_{W}=$ $-0.48, t=3.5$ and $\cos \theta=0.92$.

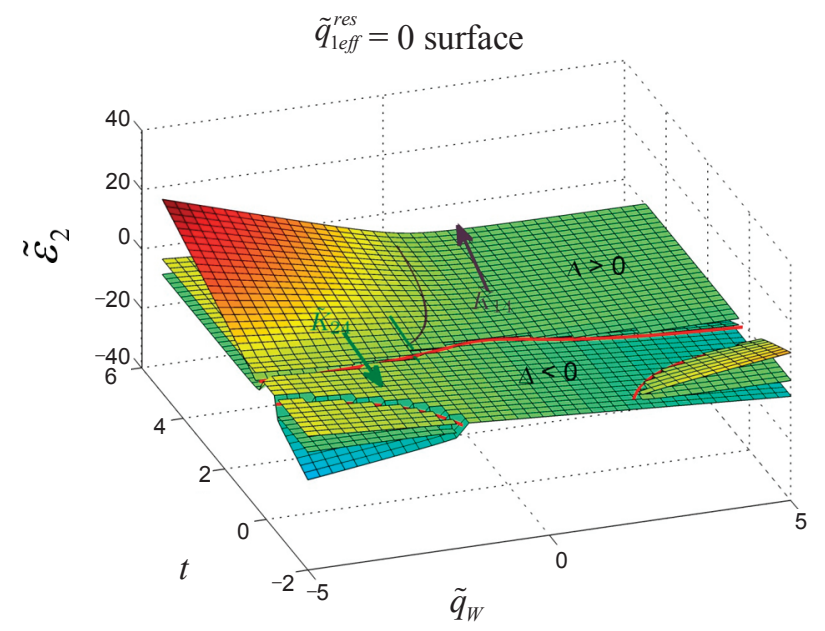

Figure 10. $\tilde{q}_{\text {leff }}^{r e s}=0$ surfaces used as a map.

ing to $\Delta=0$. One is always located at 1 independent of the value of $\tilde{q}_{W}$. Another is at 0.68 in the present case of $\tilde{q}_{W}=-1.67$. Interestingly, Martins and Zimmermann chose the value of $\tilde{K}_{24}$ corresponding to the case of multiple root, $t=1$. This choice was instrumental for them to be able to simulate the observed spectra according to the present analysis. For $t=1$, among the 3 roots, the higher two become a multiple root. For the other $t \approx 0.68$, which gives a multiple root, the lower two roots become a multiple root. Therefore, the path to the multiple root is different for the two multiple root case. Only the first type can lead to the observed spectra as will be clarified below.

Choose $t=1$ and then turn $\tilde{K}_{14}$ on progressively. As $\tilde{K}_{14}$ is 


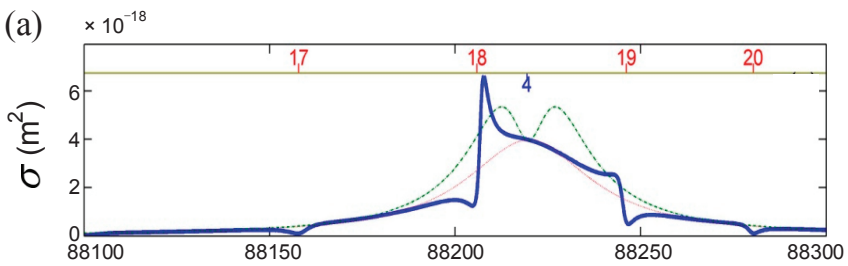

(b) $\times 10^{-18}$
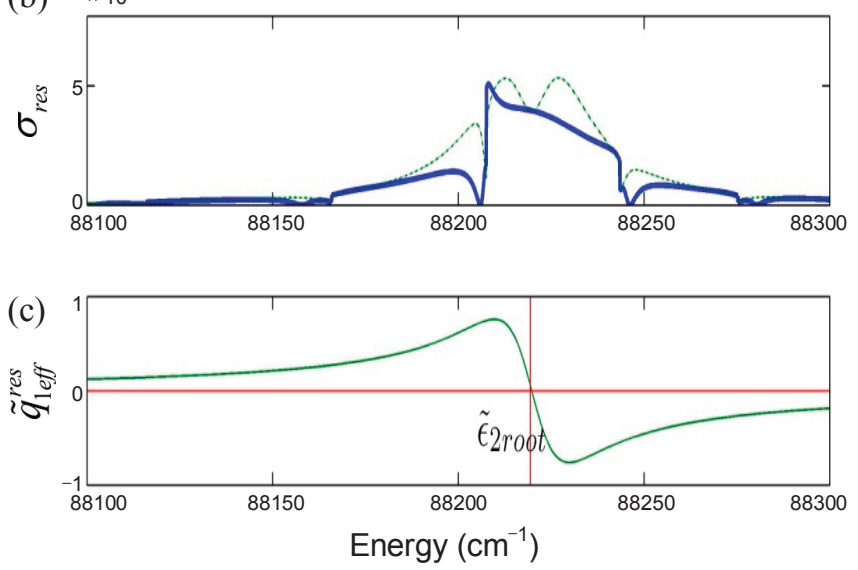

Figure 11. The same type of calculation as the one in Figs. 3 to 9 except that parameters were chosen to obtain the actual spectrum of the $\mathrm{Cu}$ I Rydberg series $3 d^{9} 4 s\left({ }^{1} D_{2}\right) n d^{2} G_{9 / 2}$ perturbed by the interloper $3 d^{9} 4 p^{2}{ }^{4} F_{9 / 2} . \tilde{K}_{12}=0, \tilde{K}_{13}=0.4, \tilde{K}_{23}=0.02, \tilde{D}_{1}=0.1, \tilde{D}_{2}=10.0$, $\tilde{D}_{3}=\tilde{D}_{4}=0$ a.u., $I_{1}=88581.0 \mathrm{~cm}^{-1}, I_{2}=128735.9 \mathrm{~cm}^{-1}, \tilde{\mu}_{1}=1.12, \tilde{\mu}_{2}=$ 0.354 a.u. are used. $\tilde{q}_{W}=0, t=1.1 \times 10^{3}$ and $\cos \theta=0.87$

turned on, the multiple root departs from each other and the one moving the lower energy pushes another root to a lower energy, as shown in Figs. 7, 8 and 9. Through this movement of the roots, the second root crosses the resonance peak corresponding to the effective quantum number $n_{1}^{*}=8$ to the energy interval between 7 and 8 , letting peaks at 7 and 8 have the opposite sign of $q$. Meanwhile, the first root moves to a lower energy, which leads to the enhancement of the resonance peak at 7 . On the other hand, the wider interval between the second and third roots makes the straddling intervening resonance more likely to occur or be more conspicuous. The routes explained so far on the zero surfaces of $\tilde{q}_{1 e f f}^{\text {res }}$ are shown in Fig. 10.

If turning $\tilde{K}_{14}$ on was not started from the multiple roots, the direction of change cannot be predicted easily and subsequent manipulation of the spectra into the desired direction may be difficult. The same type of difficulty might be expected when the second type of the multiple root was chosen.

To simulate the observed spectra much more satisfactorily, Martins and Zimmermann had to take the value of $\tilde{K}_{12}=$ zero in addition to zero transition dipole moments to open channels. The formula for this case can be obtained from Eq. (45) as follows:

$$
\tilde{q}_{1 \rho e f f}=\frac{\tilde{\varepsilon}_{2}}{\tilde{\varepsilon}_{2}^{2}+\sin ^{2} \theta}\left[-\cos ^{2} \theta+\frac{r}{r_{\rho}}\left(\tilde{\varepsilon}_{2}^{2}+1\right)\right] .
$$

Interestingly, $\tilde{q}_{1 e f f}^{r e s}$ appears to have no kinks and behave smoo- thly. This is due to the negligible value of $r$ compared to $r_{\text {res }}$ so that $\tilde{q}_{1 e f f}^{\text {res }}$ can be approximately as $\tilde{q}_{1 e f f}^{\text {res }} \approx-\tilde{\varepsilon}_{2} \cos ^{2} \theta /\left(\tilde{\varepsilon}_{2}^{2}+\right.$ $\left.\sin ^{2} \theta\right)$. In this case, the 3 roots of Eq. (46) are widely separated. Note that the small value of $r$ corresponds to the large $t$ where Fig. 10 shows the wide separation of 3 roots which was observed above for the second and third roots. The wide separation limits the value of $\tilde{q}_{1 e f f}^{r e s}$ near zero and the spectrum becomes quite smooth.

\section{Summary and Discussion}

A Rydberg series perturbed by an interloper is one of the important systems showing complex resonance phenomena due to the overlapping resonances. The reformulation of the phaseshifted MQDT initiated by Ueda to disentangle the interloper spectra from the perturbed dense Rydberg series with one open channel has been used extensively to reveal the relative influences between the interloper and dense Rydberg series caused by the overlapping of resonances. The previous formulation was naturally extended to systems involving more than 1 open channel and to determine how additional open channels affect the nature of coupling between the interloper and dense Rydberg series and eventually the observed spectra.

Although it is not difficult to reformulate the photoionization cross section formula into Ueda's type, Fano's asymmetry parameter $q$ is contaminated with the poles and is not itself useful due to the complexity caused by this contamination. This problem was reduced considerably by adopting Cohen's choice for the eigenchannels. With these eigenchannels, the troublesome poles can be sequestered into unimportant non-resonant eigenchannels leaving only kinks in the dominant resonant eigenchannels.

The fact that one eigenchannel alone shows all the resonance features suggests that such an eigenchannel acts as an effective continuum, which is sufficient to handle most of the resonance spectra eigenchannels. In other words, the eigenchannels can be made to the type of Fano's 'a, b, c,...' channels with the 'a' channel acting as an effective continuum if Cohen's convention is taken. Although Cohen's resonant eigenchannels show similar properties to Fano's effective continuum concept, their nonresonant eigenchannels are completely different from Fano's background one, in that all the singularities are sequestered into those and show very complicated energy behavior.

The present theory was applied to Martins and Zimmermann's photoionization spectra of the Rydberg series Cu I $3 d^{9} 4 s\left({ }^{1} D_{2}\right) n d$ ${ }^{2} G_{9 / 2}$ perturbed by the interloper $3 d^{9} 4 p^{24} F_{9 / 2}$ for which Cohen's 4 channel QDT failed because in the spectrum, there was a negligible direct interaction $\tilde{K}_{12}$ between the closed channels 1 and 2 compared to the indirect interaction $\tilde{K}_{13} \tilde{K}_{23}$ via open channel 3, where his theory cannot be applied. In contrast to his theory, the present theory was applied successfully to the spectrum, even with two types of simplifications, a negligible direct interaction between the closed channels and a negligible transition to the open channels, which is not available in other systems. Since the simplification available in their spectra is a generic feature of a wide class of experiments known as isolated core excitations (ICE), the theory developed for their spectra could be also applied to ICE spectra. 
In this simplification, the zero surfaces of asymmetry parameter $\tilde{q}_{1 \text { eff }}^{\text {res }}$ in the resonant eigenchannel were determined only in two parameter space. A zero surface graphic was used as a map to find the route from the starting spectra to the desired one. With the introduction of one more open channel to the 1 open 2 closed channel system, an additional incoherent process was generated and one of the poles disappeared presumably because of the release into the newly added path. Instead, one more zero surface becomes possible. Considering that $q$ zero acts as a window in the spectra, it is interesting that an additional open channel to photoionization leads to an additional window or an overall decrease in the photoionization cross section by a factor of $1+q^{2}$ compared to the nonzero asymmetry parameter $q$.

Acknowledgments. This study was supported by the Ajou university research fellowship of 2009 under contract No. 20095142COR0101S000100.

\section{Appendix A: The $\tilde{D}_{\mathbf{3}}=\mathbf{0}$ case for 3 channel QDT with 1 open} channel. The quantities containing the transition dipole moments as constituents are $\tilde{q}_{1}, \tilde{q}_{2}, \tilde{q}_{1 \text { eff }}$ and $\tilde{\sigma}_{0}$. The other quantities do not contain the transition dipole moments and are independent of the magnitudes of the transition dipole moments. They depend only on the coupling strengths. In the present case of $\tilde{D}_{3}=0, \tilde{q}_{1}$ and $\tilde{q}_{2}$ become infinite. Consider $\tilde{q}_{1 \text { eff }}$. Since the second term inside the bracket of the right side of

$$
\tilde{q}_{1 e f f}=\tilde{S}+\frac{\tilde{W}_{1}}{\tilde{W}_{1 \text { eff }}}\left[\tilde{q}_{1}-\frac{\left(\tilde{q}_{W}-\tilde{q}_{1}\right)\left(\tilde{q}_{W}-\tilde{q}_{2}\right)}{\tilde{\varepsilon}_{2}+\tilde{q}_{2}}\right]
$$

becomes $-\tilde{q}_{1}$ when $\tilde{q}_{1}$ and $\tilde{q}_{2}$ are infinite, it might be concluded incorrectly that $\tilde{q}_{\text {leff }}=\tilde{S}$. The equality of $\tilde{q}_{\text {leff }}$ with $S$ is the first order approximation, which is derived from the cancellation of the major terms. If the next order terms are considered, $\tilde{q}_{1 e f f}$ becomes

$$
\tilde{q}_{1 e f f}=S+\frac{\tilde{\varepsilon}_{2}^{2}+1}{\left(\tilde{\varepsilon}_{2}+\tilde{q}_{W}\right)^{2}}\left(\tilde{q}_{W}-\frac{\tilde{K}_{12}}{\tilde{W}_{1}} \frac{\tilde{D}_{1}}{\tilde{D}_{2}}+\frac{\tilde{\xi}_{2} \tilde{D}_{1}}{\tilde{\xi}_{1} \tilde{D}_{2}} \tilde{\varepsilon}_{2}\right)
$$

Substituting the formula for $\tilde{S}$ into (47) yields

$$
\tilde{q}_{1 e f f}=\frac{1}{\tilde{\varepsilon}_{2}+\tilde{q}_{W}}\left[\tilde{q}_{W} \tilde{\varepsilon}_{2}-1+\left(\tilde{\varepsilon}_{2}^{2}+1\right) \frac{\tilde{\xi}_{2} \tilde{D}_{1}}{\tilde{\xi}_{1} \tilde{D}_{2}}\right]
$$

Note that $\tilde{q}_{2}$ is singular when $\tilde{D}_{3}=0$ and does not act as a pole in $\tilde{q}_{\text {leff }}$. If $\tilde{D}_{1}=0$, the numerator is simplified as $\tilde{q}_{W} \tilde{\varepsilon}_{2}-1$. In this case, $\tilde{q}_{\text {leff }}$ has one simple pole at $-\tilde{q}_{W}$ and one root at $\tilde{\varepsilon}_{2}=\tilde{q}_{W}^{-1}$. If $\tilde{D}_{1} \neq 0$, the $\tilde{q}_{1 e f f}=0$ surfaces are determined by another quadratic equation

$$
r \tilde{\varepsilon}_{2}^{2}+\tilde{q}_{W} \tilde{\varepsilon}_{2}+r-1=0
$$

where $r$ is the one defined in Eq. (34).

The envelope spectrum should also be modified to avoid the singularity as follows:

$$
\tilde{\sigma}_{0 I}=\frac{4 \pi^{2} \alpha \omega}{3} \frac{\tilde{D}_{2}^{2}}{\tilde{W}_{2}\left(\tilde{\varepsilon}_{2}^{2}+1\right)}
$$

This is derived from the infinite value of $\tilde{q}_{2}$ in this case so that $\tilde{D}_{3}^{2}\left(\tilde{\varepsilon}_{2}+\tilde{q}_{2}\right)^{2}$ becomes $\tilde{D}_{3}^{2} \tilde{q}_{2}^{2}=\tilde{D}_{2}^{2} / \tilde{W}_{2}$.

\section{References}

1. Aymar, M.; Greene, C. H.; Luc-Koenig, E. Rev. Mod. Phys. 1996, 68, 1015

2. Fano, U.; Rau, A. R. P. In Atomic Collisions and Spectra; Academic: Orlando, USA, 1986; p 230.

3. Cho, B.; Lee, C.-W. Bull. Korean Chem. Soc. 2010, 31, 315.

4. Mullins, O. C.; Zhu, Y.; Xu, E. Y.; Gallagher, T. F. Phys. Rev. A $1985,32,2234$.

5. Fano, U.; Cooper, J. W. Phys. Rev. 1965, 137, A1364.

6. Mies, F. H. Phys. Rev. 1968, 175, 164.

7. Lecomte, J. M. J. Phys. B 1987, 20, 3645

8. Cohen, S. Eur. Phys. J. D 1998, 4, 31.

9. Ueda, K. Phys. Rev. A 1987, 35, 2484.

10. Martins, M.; Zimmermann, P. Z. Phys. D 1993, 27, 115.

11. Giusti-Suzor, A.; Fano, U. J. Phys. B 1984, 17, 215.

12. Cooke, W. E.; Cromer, C. L. Phys. Rev. A 1985, 32, 2725.

13. Lee, C.-W.; Kim, J. H. Bull. Korean Chem. Soc. 2002, 23, 1560.

14. Lee, C.-W. Bull. Korean Chem. Soc. 2009, 30, 891. 